\title{
Phosphorylation of NTRK1 at Y674/Y675 induced by TP53-dependent repression of PTPN6 expression: A potential novel prognostic marker for breast cancer
}

Gehad Youssef ${ }^{1,2}$, Cheryl Gillett ${ }^{3}$, Orunsola Agbaje ${ }^{4}$, Tessa Crompton ${ }^{1}$ and Ximena Montano ${ }^{1,2,5}$

${ }^{1}$ Immunobiology Unit, UCL, Institute of Child Health, London, UK; ${ }^{2}$ Molecular Hematology and Cancer Biology Unit, UCL, Institute of Child Health, London, UK; ${ }^{3}$ Breast Tissue \& Data Bank, Division of Cancer Studies, King’s College London, Guys Hospital, London, UK; ${ }^{4}$ Cancer Epidemiology Group, Division of Cancer Studies, King's College London, Guys Hospital, London, UK and ${ }^{5}$ School of Health and Social Work, Department of Allied Health Professions and Midwifery University of Hertfordshire, Hatfield, UK

We have identified a ligand-independent mechanism whereby the tumor suppressor, TP53, induces nerve growth factor receptor, NTRK1, phosphorylation at Y674/Y675 (NTRK1-pY674/pY675), via the repression of the NTRK1-phosphatase, PTPN6. This results in suppression of breast cancer cell proliferation. In this investigation, we aimed to establish whether perturbation of the wild-type TP53-NTRK1-pY674/pY675-PTPN6 pathway has an impact on disease-free survival of breast cancer patients without neo-adjuvant treatment. A total of 308 tumor samples were stained for NTRK1, NTRK1-pY674/pY675, PTPN6, and TP53 expression. Association between expression levels and disease-free survival was determined by the univariate/multivariate and Kaplan-Meir methods of analysis. DNA from tumors was sequenced to identify mutant or wild-type TP53. Tumors expressing NTRK1-pY674/pY675 but with undetectable or low levels of PTPN6 and TP53 were associated with prolonged 5,10 , and 15 years' disease-free survival by $48 \%, 36 \%$, and $37 \%$, respectively, in the multivariate analysis $(P<0.05)$. A similar result was observed in tumors expressing wild-type TP53, NTRK1pY674/pY675, and low or undetectable levels of PTPN6. Given that estrogen receptor-positive breast cancers encode wild-type TP53, we analyzed this expression pattern in these tumors. Multivariate analysis showed that it was significantly and independently predictive of prolonged survival by $66 \%, 70 \%$, and $84 \%$, respectively, $(P<0.05)$. The Kaplan-Meir method demonstrated that NTRK1-pY674/pY675 together with undetectable or low levels of PTPN6 correlated with 59\% probability of disease-free survival (median survival 15 years), compared with $7 \%$ probability of disease-free survival (median survival 4.5 years) when absent. In luminal A tumors, the presence of this pattern was estimated to have a $61 \%$ probability of disease-free survival (median survival 15 years), compared with $6 \%$ probability of disease-free survival (median survival 3 years) when it was absent. These results strongly suggest that expression of NTRK1-pY674/pY675 together with wild-type TP53 and low levels of PTPN6 expression are predictors of improved disease-free survival and that they could be useful biomarkers to predict clinical outcome.

Modern Pathology (2014) 27, 361-374; doi:10.1038/modpathol.2013.129; published online 16 August 2013

Keywords: breast cancer; NTRK1; PTPN6; TP53

Breast cancer is a leading cause of early mortality among women. ${ }^{1}$ The incidence varies worldwide,

Correspondence: Dr X Montano, PhD, Molecular Hematology and Cancer Biology Unit, UCL, Institute of Child Health, 30 Guildford Street, London WC1N 1EH, UK.

E-mail: x.montano@ucl.ac.uk

Received 12 March 2013; revised 29 May 2013; accepted 30 May 2013; published online 16 August 2013 with developed countries having higher rates than developing countries. ${ }^{1,2}$ Although the rate of breast cancer has continued to increase, ${ }^{3}$ mortality rates have decreased, partly due to the enhanced use of diagnostic and screening techniques. ${ }^{4,5}$

The nerve growth factor receptor, NTRK1, and tumor suppressor TP53 have important roles in the development of breast cancer. ${ }^{6-15}$ Nerve growth factor stimulation of NTRK1 promotes NTRK1 
activation and phosphorylation of several tyrosines (Y490, Y670, Y674, Y675, and Y785). ${ }^{15,16}$ This induces signal transduction pathways leading to proliferation, apoptosis, survival, or differentiation in a cell- or tissue-specific manner. ${ }^{15,16}$ Interestingly, in breast cancer, NTRK1 is present in all tumors, and high levels of mRNA expression are associated with good prognosis and increased patient survival. ${ }^{6}$ Paradoxically, in breast cancer cells and cell lines, nerve growth factor can stimulate NTRK1 to induce cell proliferation and metastasis ${ }^{7,8}$ and nerve growth factor expression has been associated with poor prognosis. 8,9

The tumor suppressor and transcription factor, TP53, induces differentiation, apoptosis, or cell cycle arrest by regulating transcription via protein complexes such as TBP and NF-Y. ${ }^{17-19}$ Given that TP53 has nuclear localization and exclusion signals, TP53 has nuclear and/or cytoplasmic localization. ${ }^{20}$ Interestingly, although the incidence of TP53 mutation in breast cancer is $20-30 \%,{ }^{10-13}$ tumor cells and cell lines show wild-type TP53 preferentially located in the cytoplasm, suggesting cytoplasmic sequestration as a possible mechanism of abolishing its tumor suppressor activity. ${ }^{21,22}$

PTPN6 is a tyrosine phosphatase expressed in hematopoietic and non-hematopoietic tissue and dephosphorylates receptor tyrosine kinases including CSF-1 and TCR, and receptor-associated proteins. ${ }^{23,24}$ Importantly, PTPN6 is a NTRK1-phosphatase that dephosphorylates tyrosines 674 and 675 (Y674/Y675) of NTRK1. ${ }^{25}$ In breast cancer, PTPN6 is upregulated and preferentially located in the cytoplasm of tumor cells, and high expression has been associated with poor patient prognosis. ${ }^{26,27}$

A novel mechanism for nerve growth factor-independent NTRK1 activation has been demonstrated. We have shown that in breast cancer wild-type TP53 represses PTPN6 expression via the PTPN6 proximal-P1 promoter and the NF-Y transcription factor. Repression of PTPN6 expression induces phosphorylation of NTRK1 at Y674/Y675, leading to nerve growth factor-independent NTRK1 activation. ${ }^{28}$ This signals to suppress breast cancer cell proliferation by promoting cell cycle arrest. ${ }^{28}$ These findings suggest that in breast cancer, there is a functional relationship between NTRK1, PTPN6, and wild-type TP53 involved in regulating cell division.

Given the contradictory finding that NTRK1 mRNA is associated with good prognosis whereas activation of NTRK1 protein expression by nerve growth factor induces cell proliferation, tumor growth, and metastasis, ${ }^{7,8,16}$ we asked if together expression of wild-type TP53, statistically correlates with lack of PTPN6 expression and the presence of phosphorylated NTRK1 at Y674/Y675 (NTRK1pY674/pY675), and if this expression pattern is associated with disease-free survival of breast cancer patients. Confirmation of this novel pathway could potentially lead to its use as a predictive biomarker of good prognosis and a target in drug discovery.

As most breast cancers express wild-type TP53, and as NTRK1 phosphorylation at Y674/Y675 induced by wild-type TP53-mediated PTPN6 repression could be associated with good patient outcome, the levels of NTRK1, NTRK1-pY674/pY675, PTPN6, and TP53 were assessed in 308 invasive breast cancers. Protein expression was demonstrated by immunohistochemistry. Levels of expression were correlated with disease-free survival at 5, 10, and 15 years. Results demonstrated that tumors expressing NTRK1-pY674/pY675 together with wild-type TP53 and undetectable or low levels of PTPN6 were associated with improved disease-free survival. A similar result was obtained in estrogen receptorpositive and luminal A tumors. Furthermore, the presence of NTRK1-pY674/pY675 was an independent predictor of prolonged 5, 10, and 15 years' disease-free survival by multivariate analysis when parameters were adjusted for estrogen receptor, progesterone receptor, and human epidermal growth factor receptor 2, histological grade, and metastatic node. Moreover, overexpression of PTPN6 and TP53 was associated with poor disease-free survival. These findings strongly suggest that the presence of NTRK1-pY674/pY675, wild-type TP53, and low levels of PTPN6 expression, which are involved in suppression of breast cancer cell proliferation, ${ }^{28}$ correlate with longer disease-free survival and a more favorable outcome in patients with breast cancer.

\section{Materials and methods}

\section{Tumor Samples and DNA}

A total of 308 paraffin-embedded invasive breast tumor samples, arranged in tissue microarrays were used. Three-micrometer sections from duplicate tissue microarrays containing $0.6 \mathrm{~mm}$ diameter cores were analyzed. For all samples, clinicopathological data were available (Breast Tissue and Data Bank, Guy's Hospital, UK). This included estrogen (ER), progesterone (PgR), and human epidermal growth factor 2 (HER2) receptor status. Genomic DNA from 53 matched tumors was also obtained. Approval for this work was given from the local NHS Research Ethics Committee.

\section{Immunohistochemistry}

Formalin-fixed paraffin-embedded sections were dewaxed in xylene and taken to water. Antigen retrieval was achieved using the Pascal pressure heating chamber with Dako Citrate Buffer ( $\mathrm{pH}$ 6.02) for $2 \mathrm{~min}$. The following antibodies were then applied for $40 \mathrm{~min}$ at room temperature; TP53 PAb DO-7 monoclonal antibody, 1:100, Dako, Denmark; PTPN6 polyclonal antibodies, 1:1500, Santa Cruz 
Biotechnology, USA; NTRK1 Trk-B3 antibody, 1:800 dilution, Santa Cruz Biotechnology, USA; and phosphorylated NTRK1-pY674/pY675 polyclonal antibodies, 1:50 dilution, ABgent, USA. Ki-67 was detected with monoclonal MIB-1 antibody, 1:75 dilution, Dako, Denmark. Sections were then rinsed with phosphate-buffered saline and incubated with blocking substrate buffer (Dako, Denmark). Following a further rinse, bound antibodies were detected using the Envision/HRP kit (Dako, Denmark). Sections were subsequently rinsed in tap water and counterstained with hematoxylin and then were viewed under the microscope (Leica DM LB, Germany) and images captured with a CoolSNAP Pro camera using the QCapture Pro program.

\section{Genomic DNA Sequencing}

Genomic DNA extracted from frozen tumor samples was subjected to polymerase chain reaction to amplify exons 5 and 6 , and exons 7 and 8 of the TP53 gene. High fidelity Pfu DNA polymerase with proof reading ability was used (Promega, UK). The primers for the region encoding exons 5 and 6 were: forward (5'-CTG TTC ACT TGT GCC CTG ACT TTC-3') and reverse $\left(5^{\prime}\right.$-TAG GGA GGT CAA ATA AGC AGC AGG A-3'). Primers for the region encoding exons 7 and 8 were: forward $\left(5^{\prime}\right.$-CAC TGG CCT CAT CTT GGG CCT- ${ }^{\prime}$ ) and reverse (5'-CCC CCT GAT GCA AAT GCC CCA-3'). The conditions of the polymerase chain reaction for regions encoding exons 5 and 6 , and exons 7 and 8 were: an initial step of $95^{\circ} \mathrm{C}$ for $5 \mathrm{~min}$ followed by 35 cycles using the parameters: $95^{\circ} \mathrm{C}$ for $30 \mathrm{~s}$ for the denaturation stage, an annealing stage for $40 \mathrm{~s}$ at $58{ }^{\circ} \mathrm{C}$ for the region encoding exons 5 and 6 , and $66^{\circ} \mathrm{C}$ for the region encoding exons 7 and 8 , and an elongation stage at $72{ }^{\circ} \mathrm{C}$ for $8 \mathrm{~min}$. Polymerase chain reaction was carried out using Robocycler Gradient 96 Temperature (Stratagene, UK). The amplified polymerase chain reaction products were sequenced at the National History Museum (London, UK).

\section{Statistical Analysis}

For TP53, PTPN6, NTRK1, and phosphorylated NTRK1, expression was assessed as absent, weak, moderate, or strong and denoted as $0,1,2$, and 3 . The scoring system was based on the method described by Dowsett et al. ${ }^{29} \mathrm{Ki}-67$ was scored by counting the number of positive cells out of 100 cells. Ki-67 expression present in $10 \%$ or more tumor cells was denoted as 1 and in fewer than $10 \%$ of cells was denoted as 0. Scoring was independently carried out by three of the authors (GY, XM, and CG).

The data set was stratified by hormone and growth factor receptor status, such as luminal A (ER and PgR positive, and HER2 negative) and luminal B tumors (ER and PgR positive, and HER2 positive).
Tumors were also stratified by tumor grade, ER status, and wild-type TP53 expression, respectively.

Analysis for 5-, 10-, and 15-year disease-free survival was performed to determine the prognostic significance of NTRK1-pY674/pY675 and wild-type TP53 together with low levels or absence of PTPN6 expression. Disease-free survival was defined as the time of first breast cancer diagnosis to the time of recurrence or death. Recurrent disease included; local, to the contralateral breast, and metastasis. Cumulative survival probabilities (survival function) for the different levels of expression were estimated by the Kaplan-Meir method. The Logrank test was used to determine the statistical significance of the differences between the survival functions. Univariate and multivariate analyses were used to generate a Cox regression hazard ratio model. Pearson's $\chi^{2}$ test and Fisher's exact test were used to correlate the expression of PTPN6 with histological tumor grade. A 95\% confidence interval and a $P$-value of 0.05 or less was deemed significant. The data were summarized and where appropriate, grouped when the frequency of some subcategories was low. SPSS Statistics (version 17.0) and STATA software (version 11.1) were used to perform the statistical analysis.

\section{Results}

\section{Clinicopathological Parameters and Patient Outcome}

The clinicopathological parameters of the patients and samples are described in Supplementary Table S1. Histological features were reviewed by Dr Rosemary Millis and Professor Sarah Pinder (King's College, London). None of the patients received neo-adjuvant treatment. The age of participants ranged from 45-87 years (mean $=68.0$, standard deviation $=12.5$ ). The follow-up time from the date of histological diagnosis until the date of last medical assessment or date of death had a mean time of 9.5 years (standard deviation $=6.0$ ). One-hundred and thirty-six (44\%) patients had a favorable disease-free survival outcome without recurrence, whereas 172 (56\%) patients died or had recurrence. Although, clinicopathological data are available for 308 samples, it was not possible to analyze all samples as some cores in the tissue microarrays were missing.

\section{Expression of PTPN6, TP53, Non-Phosphorylated and Phosphorylated NTRK1 at Y674/Y675, and Ki-67 in Breast Cancer Tumors}

Antibodies and immunohistochemical staining techniques of formaldehyde-fixed and paraffin-embedded tissue for NTRK1, NTRK1-pY674/pY675, PTPN6, and TP53 are well established and have been shown to be specific. ${ }^{30-38}$ The frequencies of expression intensities for NTRK1, NTRK1-pY674/ 
pY675, SHP-1, TP53, ER, HER2, PgR, and Ki-67 are summarized in Supplementary Table S2.

Given that it was important to assess the presence of NTRK1-pY674/pY675, it was critical to determine whether the breast cancer tissue samples expressed NTRK1. Figure 1 shows that NTRK1 was expressed as a punctuate pattern in the cytoplasm and the membrane as described by reports analyzing the internalization and retrograde transport of NTRK1 toward the cell bodies of neurons. ${ }^{39,40}$ Strong expression was detected in 172 (56\%) samples, whereas $84(27 \%)$ and $38(12 \%)$ samples showed moderate and weak levels, respectively. No NTRK1 expression was seen in 7 (2\%) cases (Supplementary Table S2).

The presence of NTRK1-pY674/pY675 was detected primarily in the membrane as described by other groups. ${ }^{37,38}$ Strong staining was seen in $30(13 \%)$ tumors, moderate in $53(23 \%)$, and weak in
$70(30 \%)$ samples, respectively. No NTRK1-pY674/ pY675 expression was detected in $82(35 \%)$ tumors (Supplementary Table S2).

In agreement with the previous findings, PTPN6 expression was predominantly located in the cytoplasm. ${ }^{26,27}$ High levels of cytoplasmic PTPN6 protein was detected in $30(11 \%)$ tumors, moderate in $84(29 \%)$, and weak in $112(39 \%)$ tumors. No PTPN6 was detected in $59(21 \%)$ samples (Supplementary Table S2).

TP53 was localized preferentially in the nucleus. However, cytoplasmic expression was also detected as described by former studies. ${ }^{21,22,41}$ One-hundred and twenty-four (41\%) tumors had undetectable levels of TP53 in the nucleus. TP53 was detected in the nucleus at strong, moderate, and low intensities in $33(11 \%), 46(15 \%)$, and $100(33 \%)$ cases, respectively. Two-hundred and forty-eight (82\%) had undetectable levels of TP53 in the cytoplasm.
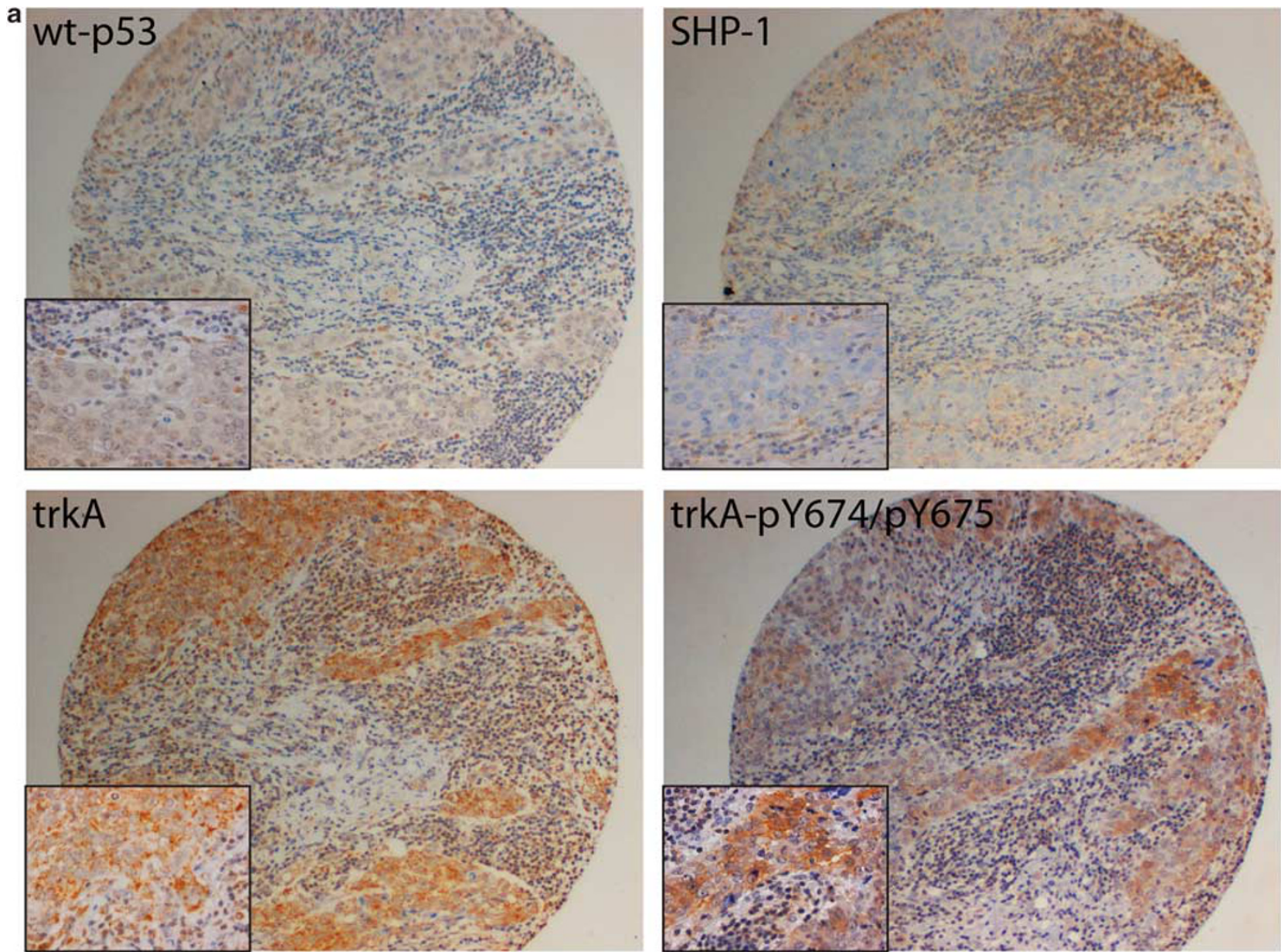

Figure 1 Immunohistochemical analysis of non-phosphorylated and phosphorylated NTRK1 at Y674/Y675, PTPN6, and TP53 in breast cancer tumor samples. (a) Detection of wild-type TP53, PTPN6, NKTRK1, and phosphorylated NTRK1 at Y674/Y675. Immunostaining of wild-type TP53 showed low levels of wild-type TP53 expression in the nucleus and cytoplasm. PTPN6 was absent or present at low levels in the cytoplasm. NTRK1 was expressed as a punctate pattern, primarily, in the cytoplasm. Phosphorylated NTRK1 at Y674/Y675 was present in the cell membrane. (b) Detection of mutant TP53, PTPN6, NTRK1, and phosphorylated NTRK1 at Y674/Y675. Strong expression of mutant TP53 was observed in the nucleus. Strong levels of PTPN6 were detected in the cytoplasm. NTRK1 was expressed as a punctate pattern, primarily, in the cytoplasm. Phosphorylated NTRK1 at Y674/Y675 was not detected in the cell membrane. Photographs were taken at $\times 10$ and insert at $\times 40$ magnification, respectively using a Leica microscope. 

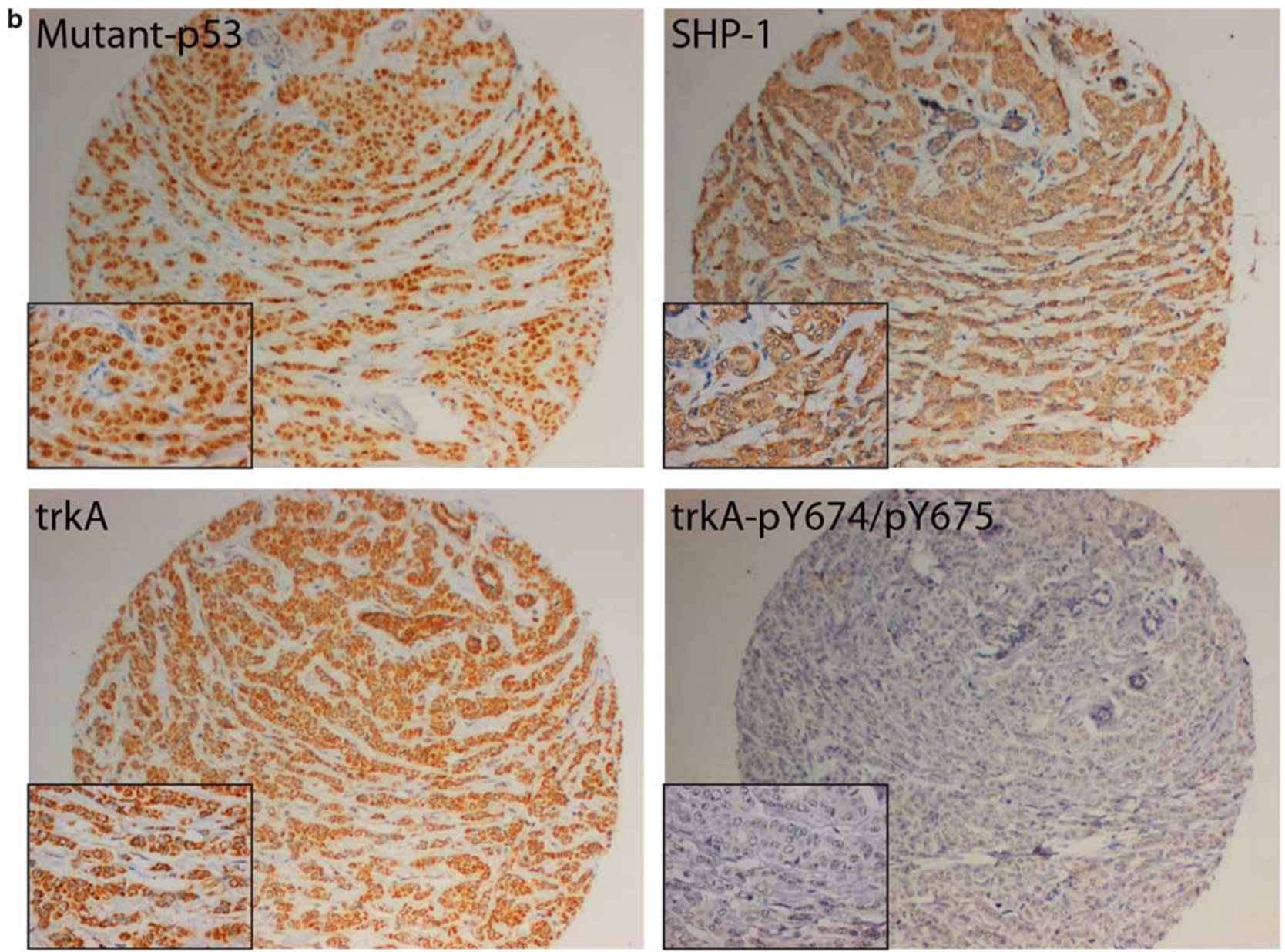

Figure 1 Continued.

There was a lack of tumors with strong cytoplasmic TP53 expression; however, 42 (14\%) had moderate expression and $12(4 \%)$ had weak levels. In 111 cases, TP53 was not detected together in the nucleus and cytoplasm (Supplementary Table S2).

Importantly, NTRK1-pY674/pY675 was observed in tumors that expressed low or undetectable TP53 and PTPN6 (Figure 1a) and was not seen in tumors that expressed high levels of these two proteins (Figure 1b). These results suggest that NTRK1pY674/pY675 together with low or undetected levels of TP53 and PTPN6 can be present in breast tumor samples.

\section{Association of PTPN6, TP53, and Phosphorylated NTRK1 at Y674/Y675 with Disease-free Survival}

To investigate whether expression of NTRK1pY674/pY675 and low or undetectable levels of PTPN6 and TP53 could have prognostic significance, multivariate analysis adjusted for ER, HER2, PgR, node status, and Ki-67 expression was performed for 5, 10, and 15 years' disease-free survival (Table 1). Patients whose tumors had any level of
NTRK1-pY674/pY675 expression showed significantly improved survival compared with those who had none by $67 \%$ (hazard ratio $=0.33$, $P=0.003$ ), $53 \%$ (hazard ratio $=0.47, P=0.013$ ), and $48 \%$ (hazard ratio $=0.52, P=0.037$ ), respectively. Samples with strong PTPN6 expression had hazard ratios of $1.53,1.19$, and 1.25 , respectively, suggestive of an association with shorter diseasefree survival. However, the $P$-values were greater than 0.05. Moderate cytoplasmic TP53 expression was associated with shorter 5-, 10-, and 15-year disease-free survival with hazard ratios of 1.90 $(P=0.296), 2.94 \quad(P=0.033)$, and $3.24 \quad(P=0.016)$, respectively. Patients with strong levels of nuclear TP53 in their tumor showed a trend toward shorter disease-free survival with hazard ratios of 1.61, 1.35, and 1.31 , respectively; however, the $P$-values were greater than 0.05 . These results suggest that the presence of NTRK1-pY674/pY675 is associated with longer disease-free survival and overexpression of PTPN6 and TP53 is associated with reduced disease-free survival.

In order to assess whether our variables remained significant in the presence of other key histological 
Table 1 Multivariate analysis ${ }^{a}$ of 308 tumor samples for 5-, 10-, and 15-year disease-free survival

\begin{tabular}{|c|c|c|c|c|c|c|}
\hline \multirow[b]{2}{*}{ Variable } & \multicolumn{2}{|c|}{ 5-year } & \multicolumn{2}{|c|}{ 10-year } & \multicolumn{2}{|c|}{ 15-year } \\
\hline & Hazard ratio (CI) & $\mathrm{P}$-value & Hazard ratio (CI) & $\mathrm{P}$-value & Hazard ratio (CI) & $\mathrm{P}$-value \\
\hline \multicolumn{7}{|c|}{ NTRK1-pY674/pY675 } \\
\hline 0 & 1 & & 1 & & 1 & \\
\hline 1 & $0.81(0.39-1.66)$ & 0.566 & $0.87(0.48-1.60)$ & 0.659 & $0.83(0.47-1.47)$ & 0.522 \\
\hline $2 / 3$ & $0.33(0.16-0.68)$ & 0.003 & $0.47(0.26-0.85)$ & 0.013 & $0.52(0.33-0.97)$ & 0.037 \\
\hline \multicolumn{7}{|l|}{ PTPN6 } \\
\hline $0 / 1 / 2$ & 1 & & 1 & & 1 & \\
\hline 3 & $1.53(0.69-3.42)$ & 0.288 & $1.19(0.58-2.46)$ & 0.638 & $1.25(0.66-2.37)$ & 0.493 \\
\hline \multicolumn{7}{|c|}{ TP53 (cytoplasm) } \\
\hline 0 & 1 & & 1 & & 1 & \\
\hline 1 & $2.36(1.18-6.07)$ & 0.065 & $1.44(0.64-3.25)$ & 0.377 & $1.66(0.84-3.27)$ & 0.146 \\
\hline 2 & $1.90(0.57-6.35)$ & 0.296 & $2.94(1.09-7.95)$ & 0.033 & $3.24(1.25-8.86)$ & 0.016 \\
\hline \multicolumn{7}{|c|}{ TP53 (nucleus) } \\
\hline 0 & 1 & & 1 & & 1 & \\
\hline 1 & $0.59(0.26-1.36)$ & 0.214 & $0.56(0.29-1.07)$ & 0.081 & $0.81(0.47-1.41)$ & 0.460 \\
\hline $2 / 3$ & $1.61(0.78-3.32)$ & 0.196 & $1.35(0.74-2.45)$ & 0.321 & $1.31(0.74-2.31)$ & 0.354 \\
\hline \multicolumn{7}{|l|}{ ER status } \\
\hline 0 & 1 & & 1 & & 1 & \\
\hline 1 & $0.59(0.28-1.23)$ & 0.158 & $0.73(0.39-1.36)$ & 0.325 & $0.88(0.49-1.57)$ & 0.655 \\
\hline \multicolumn{7}{|c|}{ HER2 status } \\
\hline 0 & 1 & & 1 & & 1 & \\
\hline 1 & $2.16(1.03-4.51)$ & 0.041 & $1.61(0.87-2.97)$ & 0.129 & $1.60(0.91-2.81)$ & 0.104 \\
\hline \multicolumn{7}{|l|}{ PgR status } \\
\hline 0 & 1 & & 1 & & 1 & \\
\hline 1 & $0.95(0.50-1.78)$ & 0.893 & $0.90(0.54-1.51)$ & 0.684 & $1.02(0.64-1.63)$ & 0.940 \\
\hline \multicolumn{7}{|l|}{ Node status } \\
\hline Negative & 1 & & 1 & & 1 & \\
\hline Positive & $1.91(1.02-3.56)$ & 0.042 & $1.98(1.20-3.29)$ & 0.008 & $1.86(1.18-2.93)$ & 0.007 \\
\hline \multicolumn{7}{|l|}{$\mathrm{Ki}-67(\%)$} \\
\hline$<10$ & 1 & & 1 & & 1 & \\
\hline$>10$ & $1.97(0.88-4.39)$ & 0.097 & $1.84(0.97-3.50)$ & 0.062 & $1.73(0.97-3.07)$ & 0.061 \\
\hline
\end{tabular}

CI, confidence interval.

${ }^{\mathrm{a}}$ Multivariate analysis Cox regression model. 0, absence of expression; 1, weak expression; 2, moderate expression; 3, strong expression. Bold values indicate significant $P$-values.

biomarkers, we included ER (a good predictive marker ${ }^{42,43}$ ), PgR (a marker that is considered to participate with ER to provide an additional positive prediction in ER-positive tumors ${ }^{43,44}$ ), and HER2 status (a marker of poor predictive outcome ${ }^{45,46}$ ) in the multivariate analysis (Table 1). The presence of ER showed a trend toward improved 5, 10, and 15 years' disease-free survival by $41 \%$ (hazard ratio = $0.59, \quad P=0.158$ ), $27 \% \quad$ (hazard ratio $=0.73$, $P=0.325$ ), and $12 \%$ (hazard ratio $=0.88$, $P=0.655$ ), respectively. Whereas, and as expected, HER2-positive status was associated with reduced disease-free survival with hazard ratios of 2.16 $(P=0.041), 1.61 \quad(P=0.129)$, and $1.60 \quad(P=0.104)$, respectively. PgR expression showed a modest trend toward improved disease-free survival with hazard ratios of $0.95,0.90$, and 1.02 , respectively. Also, the presence of one or more positive axillary nodes was associated with poor disease-free survival with increased hazard ratios of $1.91(P=0.042), 1.98$ $(P=0.008)$, and $1.86(P=0.007)$.

Elevated PTPN6 expression has been shown to be associated with high tumor grade. ${ }^{27}$ Interestingly, when histological grade was included in the multivariate analysis, the hazard ratios of PTPN6 were perturbed (Supplementary Table S3). The hazard ratio of 5,10 , and 15 years' disease-free survival was reduced from 1.54 to $1.35,1.19$ to 0.97 , and 1.25 to 1.01 , respectively, which is indicative of a positive correlation between histological grade and PTPN6 expression. These findings are further supported by the Spearman's rank test (rank $=0.151$, $P=0.015$ ) and Pearson's $\chi^{2}$ test (correlation $=0.153$, $P=0.013$ ), which show that high levels of PTPN6 correlate with high histological grade. Importantly, as shown in Supplementary Table S4, when the data set is stratified for grade III tumors, high PTPN6 expression showed a trend toward a lower 
Table 2 Multivariate analysis ${ }^{a}$ of tumor samples expressing phosphorylated NTRK1 at Y674/Y675 together with lack or low levels of TP53 and PTPN6

\begin{tabular}{|c|c|c|c|c|c|c|}
\hline \multirow[b]{2}{*}{ Variable } & \multicolumn{2}{|c|}{ 5-year } & \multicolumn{2}{|c|}{ 10-year } & \multicolumn{2}{|c|}{ 15-year } \\
\hline & Hazard ratio (CI) & P-value & Hazard ratio (CI) & P-value & Hazard ratio (CI) & $\mathrm{P}$-value \\
\hline \multicolumn{7}{|c|}{ NTRK1-pY674/pY675/PTPN6/TP53 mechanism } \\
\hline Fails & 1 & & 1 & & 1 & \\
\hline Meets $^{\mathrm{b}}$ & $0.52(0.30-0.90)$ & 0.021 & $0.64(0.41-0.99)$ & 0.049 & $0.63(0.42-0.95)$ & 0.029 \\
\hline \multicolumn{7}{|l|}{ ER status } \\
\hline 0 & 1 & & 1 & & 1 & \\
\hline 1 & $0.59(0.31-1.10)$ & 0.098 & $0.82(0.48-1.40)$ & 0.462 & $0.96(0.58-1.60)$ & 0.877 \\
\hline \multicolumn{7}{|l|}{ HER2 status } \\
\hline 0 & 1 & & 1 & & 1 & \\
\hline 1 & $2.15(1.15-4.02)$ & 0.017 & $1.76(1.03-2.99)$ & 0.038 & $1.59(0.97-2.61)$ & 0.065 \\
\hline \multicolumn{7}{|l|}{ PgR status } \\
\hline 0 & 1 & & 1 & & 1 & \\
\hline 1 & $1.22(0.69-2.17)$ & 0.493 & $1.03(0.64-1.63)$ & 0.915 & $1.05(0.69-1.61)$ & 0.815 \\
\hline \multicolumn{7}{|l|}{ Node status } \\
\hline Negative & 1 & & 1 & & 1 & \\
\hline Positive & $1.80(1.03-3.14)$ & 0.041 & $2.05(1.29-3.26)$ & 0.002 & $1.80(1.19-2.73)$ & 0.006 \\
\hline \multicolumn{7}{|l|}{$K i-67(\%)$} \\
\hline$<10$ & 1 & & 1 & & 1 & \\
\hline$>10$ & 1.99 (1.02-3.88) & 0.044 & $1.96(1.14-3.38)$ & 0.015 & $2.04(1.24-3.37)$ & 0.005 \\
\hline
\end{tabular}

CI, confidence interval.

${ }^{a}$ Multivariate analysis Cox regression model. o, absence of expression; 1; weak expression; 2, moderate expression; 3, strong expression.

b Meets: Presence of phosphorylated NTRK1 at Y674/Y675 and absence or low expression of TP53 and PTPN6.

Bold values indicate significant $P$-values.

disease-free survival when correlated to the unstratified data set, with hazard ratios of $2.44(P=0.094)$, $2.11(P=0.123)$, and $2.36(P=0.055)$, respectively.

Interestingly, the hazard ratios of NTRK1-pY674/ pY675 were not affected when histological grade was included in the multivariate analysis. This suggests that NTRK1-pY674/pY675 is an independent predictor of improved disease-free survival regardless of whether histological grade is included in the multivariate analysis (Supplementary Figure S3).

Wild-type TP53 is expressed at low or undetectable levels in breast cancer cells. ${ }^{4-50}$ Thus, in order to determine whether NTRK1-pY674/pY675 together with undetectable or low levels of PTPN6 and TP53 expression are associated with improved disease-free survival, tumors showing together this NTRK1-pY674/pY675, PTPN6, and TP53 expression pattern were analyzed as they meet the conditions described by Montano. ${ }^{28}$ Tumors meeting these conditions were denoted as 1 and others were denoted as 0. As seen in Table 2, multivariate analysis showed this expression pattern to be an independent predictor of improved 5-, 10-, and 15-year disease-free survival by $48 \%$ (hazard ratio $=0.52$, $P=0.021$ ), $36 \%$ (hazard ratio $=0.64, P=0.049$ ), and $37 \%$ (hazard ratio $=0.63, P=0.029$ ) when ER, HER2, PgR, nodal status, and Ki-67 expressions were included. From 308 samples, this pattern was present in 135 and absent in 130 samples. Information was not available for 40 cases. These results strongly suggest that reduced or lack of PTPN6 and TP53 levels, together with the presence of NTRK1-pY674/ pY675 are associated with longer disease-free survival.

\section{Analysis of TP53 Gene and Correlation with Phosphorylated NTRK1 at Y674/Y675}

Given that immunohistochemical staining of TP53 does not differentiate between wild-type and mutant forms of the gene, exons 5-8 were sequenced. These were chosen, as they undergo a high frequency of mutations. ${ }^{11-13}$ DNA sequencing was performed in polymerase chain reaction-amplified regions from genomic DNA from 53 available tumor samples. Wild-type and mutant TP53 were present in $45(85 \%)$ and $8(15 \%)$ samples, respectively, which is consistent with the previous studies reporting mutation frequencies in breast cancer tumors of $20-30 \% .{ }^{11-13,51-53}$ Univariate analysis was performed for 3 and 5 years' disease-free survival. As only 45 tumors expressed wild-type TP53, it was not possible to undertake the analysis for 10 and 15 years' disease-free survival. Univariate analysis (Supplementary Table S5) revealed that wild-type TP53 and NTRK1-pY674/pY675 had a trend toward improved 3- and 5-year disease-free survival by $84 \%$ (hazard ratio $=0.16$ ) and $73 \%$ (hazard ratio $=0.27$ ), respectively; however, the $P$-values were greater 
than 0.05. This is most likely due to the low DNA sample number available for the analysis. As PTPN6 overexpression was observed in only six cases in the stratified data set, it was not possible to analyze its expression by univariate analysis. These results suggest that there is a trend where wild-type TP53 expression is associated with the presence of NTRK1-pY674/pY675.

In order to explore whether tumors that express wild-type TP53 together with NTRK1-pY674/pY675 and low or lack of PTPN6 expression are associated with favorable disease-free survival, univariate analysis for tumors expressing wild-type TP53 and low or lack of PTPN6 levels together with NTRK1pY674/pY675 was carried out. As seen in Supplementary Table S5, results showed a trend toward good 3 and 5 years' disease-free survival by $41 \%$ (hazard ratio $=0.59, \quad P=0.425$ ) and $29 \%$ (hazard ratio $=0.71, P=0.557$ ), respectively. Although the $P$-values were greater than 0.05 , these findings suggest that in wild-type TP53 encoding tumors, NTRK1-pY674/pY675 and PTPN6 trends in a similar way to the analysis performed in the full database shown in Table 2.

\section{Phosphorylated NTRK1 at Y674/Y675 and PTPN6 Expression in Estrogen Receptor-Positive Tumors}

Reports have established that estrogen receptor expression strongly correlates with the presence of wild-type TP53 in breast cancer tumors. ${ }^{42,54,55}$ Based on this key finding and given that DNA from only 53 samples was sequenced, it was inferred that estrogen receptor-positive tumors in our data set expressed wild-type TP53.

Multivariate analysis (Table 3) adjusted for HER2, PgR, node status, and Ki-67 identified NTRK1pY674/pY675 as an independent predictor of improved 5-, 10-, and 15-year disease-free survival by $77 \%$ (hazard ratio $=0.23, P=0.001$ ), $66 \%$ (hazard ratio $=0.34, P=0.002$ ), and $54 \%$ (hazard ratio $=0.46, \quad P=0.013$ ), respectively. Elevated PTPN6 expression, independently, predicted poor survival with increased hazard ratios of 2.60 $(P=0.029), 2.07 \quad(P=0.069)$, and $2.13(P=0.040)$. In the case of HER2, there was a trend for reduced disease-free survival with hazard ratios of 2.19, 1.54, and 1.43 respectively. However, HER2 did not independently predict poor survival outcome $(p>0.05)$. Moreover, and as expected, nodal status was an independent predictor of poor survival with hazard ratios of $2.83(P=0.008), 2.61(P=0.002)$, and $2.31(P=0.002)$, respectively. These results suggest that in estrogen receptor-positive tumors, NTRK1-pY674/pY675 expression is associated with improved disease-free survival, whereas elevated PTPN6 expression is associated with poor diseasefree survival. HER2, high tumor grade, and the presence of metastatic nodes also correlate with reduced disease-free survival. Overall, these results suggest that in estrogen receptor-positive tumors,

Table 3 Multivariate analysis ${ }^{a}$ of estrogen receptor-positive samples for 5-, 10- and 15-year disease-free survival

\begin{tabular}{|c|c|c|c|c|c|c|}
\hline \multirow[b]{2}{*}{ Variable } & \multicolumn{2}{|c|}{ 5-year } & \multicolumn{2}{|c|}{ 10-year } & \multicolumn{2}{|c|}{ 15-year } \\
\hline & Hazard ratio (CI) & $\mathrm{P}$-value & Hazard ratio (CI) & $\mathrm{P}$-value & Hazard ratio (CI) & $\mathrm{P}$-value \\
\hline \multicolumn{7}{|c|}{ NTRK1-pY674/pY675 } \\
\hline 0 & 1 & & 1 & & 1 & \\
\hline 1 & $0.53(0.23-1.22)$ & 0.137 & $0.65(0.33-1.28)$ & 0.217 & $0.68(0.36-1.28)$ & 0.230 \\
\hline $2 / 3$ & $0.23(0.10-0.54)$ & 0.001 & $0.34(0.17-0.68)$ & 0.002 & $0.46(0.25-0.85)$ & 0.013 \\
\hline \multicolumn{7}{|l|}{ PTPN6 } \\
\hline $0 / 1 / 2$ & 1 & & 1 & & 1 & \\
\hline 3 & $2.60(1.10-6.13)$ & 0.029 & $2.07(0.95-4.55)$ & 0.069 & $2.13(1.03-4.39)$ & 0.040 \\
\hline \multicolumn{7}{|l|}{ HER2 status } \\
\hline 0 & 1 & & 1 & & 1 & \\
\hline 1 & $2.19(1.00-4.82)$ & 0.050 & $1.54(0.80-2.98)$ & 0.196 & $1.43(0.78-2.62)$ & 0.244 \\
\hline \multicolumn{7}{|l|}{ PgR status } \\
\hline 0 & 1 & & 1 & & 1 & \\
\hline 1 & $0.93(0.45-1.91)$ & 0.841 & $0.97(0.53-1.76)$ & 0.913 & $1.15(0.66-1.98)$ & 0.624 \\
\hline \multicolumn{7}{|l|}{ Ki-67(\%) } \\
\hline$<10$ & 1 & & 1 & & 1 & \\
\hline$>10$ & $2.24(0.87-5.75)$ & 0.094 & $1.97(0.93-4.18)$ & 0.078 & $1.69(0.87-3.27)$ & 0.123 \\
\hline \multicolumn{7}{|l|}{ Node status } \\
\hline Negative & 1 & & 1 & & 1 & \\
\hline Positive & $2.83(1.31-6.09)$ & 0.008 & $2.61(1.42-4.79)$ & 0.002 & $2.31(1.35-3.95)$ & 0.002 \\
\hline
\end{tabular}

CI, confidence interval.

${ }^{\mathrm{a}}$ Multivariate analysis Cox regression model. 0, absence of expression; 1, weak expression 2, moderate expression 3, strong expression. Bold values indicate significant $P$-values. 
Table 4 Multivariate analysis ${ }^{a}$ of estrogen receptor-positive samples expressing phosphorylated NTRK1 at Y674/Y675 together with lack or low levels of TP53 and PTPN6

\begin{tabular}{|c|c|c|c|c|c|c|}
\hline \multirow[b]{2}{*}{ Variable } & \multicolumn{2}{|c|}{ 5-year } & \multicolumn{2}{|c|}{ 10-year } & \multicolumn{2}{|c|}{ 15-year } \\
\hline & Hazard ratio (CI) & $\mathrm{P}$-value & Hazard ratio (CI) & $\mathrm{P}$-value & Hazard ratio (CI) & $\mathrm{P}$-value \\
\hline \multicolumn{7}{|c|}{ NTRK1-pY674/pY675/PTPN6/TP53 mechanism } \\
\hline Fails & 1 & & 1 & & 1 & \\
\hline Meets $^{\mathrm{b}}$ & $0.34(0.17-0.67)$ & 0.002 & $0.30(0.17-0.51)$ & $<0.001$ & $0.24(0.16-0.52)$ & $<0.001$ \\
\hline \multicolumn{7}{|c|}{ HER2 status } \\
\hline 0 & 1 & & 1 & & 1 & \\
\hline 1 & $1.57(0.77-3.22)$ & 0.217 & $1.24(0.68-2.26)$ & 0.477 & $1.03(0.84-2.25)$ & 0.927 \\
\hline \multicolumn{7}{|l|}{ PgR status } \\
\hline 0 & 1 & & 1 & & 1 & \\
\hline 1 & $0.76(0.38-1.53)$ & 0.446 & $0.77(0.44-1.34)$ & 0.355 & $0.81(0.62-1.50)$ & 0.425 \\
\hline \multicolumn{7}{|l|}{$\mathrm{Ki}-67(\%)$} \\
\hline$<10$ & 1 & & 1 & & 1 & \\
\hline$>10$ & $1.13(0.50-2.57)$ & 0.775 & $1.47(0.74-2.92)$ & 0.274 & $1.75(0.98-2.86)$ & 0.080 \\
\hline \multicolumn{7}{|c|}{ Node status } \\
\hline Negative & 1 & & 1 & & 1 & \\
\hline Positive & $2.21(1.10-4.45)$ & 0.026 & $2.37(1.35-4.17)$ & 0.003 & $1.97(1.16-2.77)$ & 0.007 \\
\hline \multicolumn{7}{|c|}{ Histological grade } \\
\hline I & 1 & & 1 & & 1 & \\
\hline II & $3.19(0.70-14.4)$ & 0.132 & $3.93(1.16-13.3)$ & 0.028 & $2.42(0.86-3.42)$ & 0.039 \\
\hline III & $4.91(1.09-22.2)$ & 0.039 & $5.16(1.50-17.7)$ & 0.009 & $2.68(1.12-4.41)$ & 0.025 \\
\hline
\end{tabular}

CI, confidence interval.

${ }^{\mathrm{a}}$ Multivariate analysis Cox regression model. 0, absence of expression; 1, weak expression 2, moderate expression; 3, strong expression.

${ }^{b}$ Meets: Presence of phosphorylated NTRK1 at Y674/Y675 and absence or low expression of TP53 and PTPN6.

Bold values indicate significant $P$-values.

NTRK1-pY674/pY675 and low or lack of PTPN6 levels are independent predictors of 5,10 , and 15 years' disease-free survival.

\section{Phosphorylated NTRK1 at Y674/Y675 and PTPN6 Expression in Luminal A Tumors}

Breast cancer tumors have been historically classified based on histological tumor type. A potentially more refined type of classification based on ER, PgR, and HER2 has emerged. Although different groups have used a variation of receptor combinations to identify tumor subtypes, the general consensus is that tumors can be classified as: luminal A (ER and PgR positive, HER2 negative), luminal B (ER and PgR positive, HER2 positive), triple negative (ER, PgR and HER2 negative), and HER2 positive (ER and PgR negative, HER2 positive). ${ }^{55-59}$ These studies have also shown that in a given population, the incidence of luminal A tumors is $48-58 \%$, luminal B tumors is $19-25 \%$, triple negative tumors is $11-$ $23 \%$, and HER2-positive tumors is $5-11 \% .{ }^{55-59}$

Based on this type of classification, analysis of the frequencies of the sub-groups in our data set has shown that $129(50 \%)$ are luminal A, $75(29 \%)$ are luminal B, $37(14 \%)$ are triple negative, and $15(6 \%)$ are HER2-positive tumors. There was insufficient information for 52 samples to classify them into one of the sub-groups, as either ER, PgR, or HER2 expression data were not available.

As luminal A tumors are associated with good clinical outcome ${ }^{57,58}$ and presence of wild-type TP53, ${ }^{60}$ multivariate analysis was carried out for NTRK1-pY674/pY675 and PTPN6 expression. As shown in Table 4, results revealed that NTRK1pY674/pY675 was associated with improved 5, 10 , and 15 years' disease-free survival by $65 \%$ (hazard ratio $=0.35, P=0.056$ ), $59 \%$ (hazard ratio $=$ $0.41, P=0.039$ ), and $54 \%$ (hazard ratio $=0.46$, $P=0.039$ ), respectively. High levels of PTPN6 trended toward a lower disease-free survival by $2 \%$ (hazard ratio $=1.02$ ), $63 \%$ (hazard ratio $=1.63$ ), and $64 \%$ (hazard ratio $=1.64$ ); however, the $P$-values were greater than 0.05 . These results suggest that in luminal A tumors, NTRK1-pY674/pY675 is associated with good disease-free survival. Based on the a5nalysis of PTPN6 expression, it can be inferred that undetectable and low levels of PTPN6 are also associated with improved disease-free survival.

\section{Expression of Phosphorylated NTRK1 at Y674/Y675 together with Low or Lack of PTPN6 Expression in Estrogen Receptor-Positive Tumors}

In order to statistically correlate together NTRK1pY674/pY675, and lack of PTPN6 and wild-type 
Table 5 Multivariate analysis ${ }^{\mathrm{a}}$ of NTRK1 phosphorylation at Y674/Y675 and PTPN6 of luminal $\mathrm{A}^{\mathrm{b}}$ breast cancer tumors

\begin{tabular}{|c|c|c|c|c|c|c|}
\hline \multirow[b]{2}{*}{ Variable } & \multicolumn{2}{|c|}{5 -year } & \multicolumn{2}{|c|}{ 10-year } & \multicolumn{2}{|c|}{ 15-year } \\
\hline & Hazard ratio (CI) & $\mathrm{P}$-value & Hazard ratio (CI) & $\mathrm{P}$-value & Hazard ratio (CI) & $\mathrm{P}$-value \\
\hline \multicolumn{7}{|c|}{ NTRK1-pY674/pY675 } \\
\hline 0 & 1 & & 1 & & 1 & \\
\hline 1 & $0.65(0.24-1.77)$ & 0.398 & $0.69(0.31-1.53)$ & 0.365 & $0.72(0.35-1.47)$ & 0.368 \\
\hline $2 / 3$ & $0.35(0.12-1.03)$ & 0.056 & $0.41(0.18-0.96)$ & 0.039 & $0.46(0.22-0.96)$ & 0.039 \\
\hline \multicolumn{7}{|l|}{ PTPN6 } \\
\hline $0 / 1 / 2$ & 1 & & 1 & & 1 & \\
\hline 3 & $1.02(0.24-4.40)$ & 0.976 & $1.63(0.63-4.24)$ & 0.312 & $1.64(0.69-3.91)$ & 0.262 \\
\hline
\end{tabular}

CI, confidence interval.

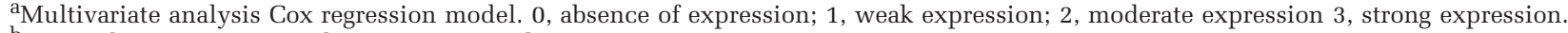

bLuminal A tumors, ER and PgR positive and HER2 negative.

Bold values indicate significant $P$-values.

TP53 expression in estrogen receptor-positive tumors, a multivariate analysis adjusted for tumor grade, metastatic nodal status, HER2, PgR, and Ki-67 was undertaken. As indicated previously, it was reasoned that estrogen receptor-positive tumors code for wild-type TP53. As shown in Table 5, results revealed that together NTRK1-pY674/pY675 and undetectable or low levels of PTPN6 and TP53 were associated with improved 5-, 10-, and 15-year disease-free survival by $66 \%$ (hazard ratio $=0.34$, $P=0.002$ ), $70 \%$ (hazard ratio $=0.30, P<0.001$ ), and $76 \%$ (hazard ratio $=0.24, P<0.001$ ), respectively. Of the adjusted parameters, only node status was an independent predictor of survival with hazard ratios of $2.21 \quad(P=0.026), \quad 2.37 \quad(P=0.003)$, and 1.97 $(P=0.007)$, respectively. Using the Kaplan-Meir method (Figure 2a), it was estimated that tumors with this expression pattern had 59\% probability of 15 years disease-free survival with a median survival time of over 15 years. Whereas in its absence, the chance of survival was reduced to $7 \%$ with a median survival time of 4.5 years. This difference was statistically significant $(P<0.001)$ as identified by Log-rank analysis. These results strongly suggest that in estrogen receptor-positive tumors, the presence of NTRK1-pY674/pY675 and lack or low levels of PTPN6 is associated with improved disease-free survival. Giving that it was reasoned that estrogen receptor-positive tumors express wild-type TP53, these results suggest that together wild-type TP53, NTRK1-pY674/pY675, and low or lack of PTPN6 expression are associated with improved disease-free survival.

\section{Expression of Phosphorylated NTRK1 at Y674/Y675 together with Wild-Type TP53 and Low or Lack of PTPN6 Expression in Luminal A Tumors}

In order to assess the relationship between NTRK1pY674/pY675, PTPN6 and wild-type TP53 in luminal A tumors, a similar analysis to the one conducted with estrogen receptor-positive tumors was undertaken. As shown in Table 6, in luminal A tumors, analysis of NTRK1-pY674/pY675 together with low or lack of PTPN6 expression revealed a significant association with improved 5, 10, and 15 years' disease-free survival by $71 \%$ (hazard ratio $=0.29, P=0.008$ ), $75 \%$ (hazard ratio $=0.25$, $P=<0.001$ ), and $79 \%$ (hazard ratio $=0.21$, $P=<0.001)$, respectively. There was a $61 \%$ probability of survival in the presence of these variables compared with only $6 \%$ survival in its absence $(P<0.001$, Figure $2 \mathrm{~b})$. The median survival time was 15 years in tumors where this protein expression pattern was present compared with 3 years in samples where it was absent. These results strongly suggest that NTRK1-pY674/pY675 together with low or lack of PTPN6 expression in luminal A tumors provide an independent and additional prediction factor of survival outcome. Moreover, as luminal A tumors express wild-type TP53, ${ }^{58,60}$ from these results it can be inferred that together wild-type TP53, NTRK1-pY674/pY675, and low or lack of PTPN6 expression are associated with improved disease-free survival.

\section{Discussion}

Results show that the presence of NTRK1-pY674/ pY675 together with undetectable or low PTPN6 and TP53 expression is associated with improved 5-, 10-, and 15-year disease-free survival in this study of 308 patients. This is the first investigation that associates NTRK1-pY674/pY675 with disease-free survival in the context of wild-type TP53 and undetectable or low expression of PTPN6. Importantly, NTRK1pY674/pY675 was predictive of longer disease-free survival, whereas PTPN6 and TP53 overexpressions were predictive of reduced disease-free survival. These results were also seen in estrogen receptorpositive and luminal A tumors. These findings are significant, as they demonstrate that components of this novel mechanism of NTRK1-Y674/Y675 phosphorylation $^{28}$ offer a potential target for therapy for breast cancer patients. 

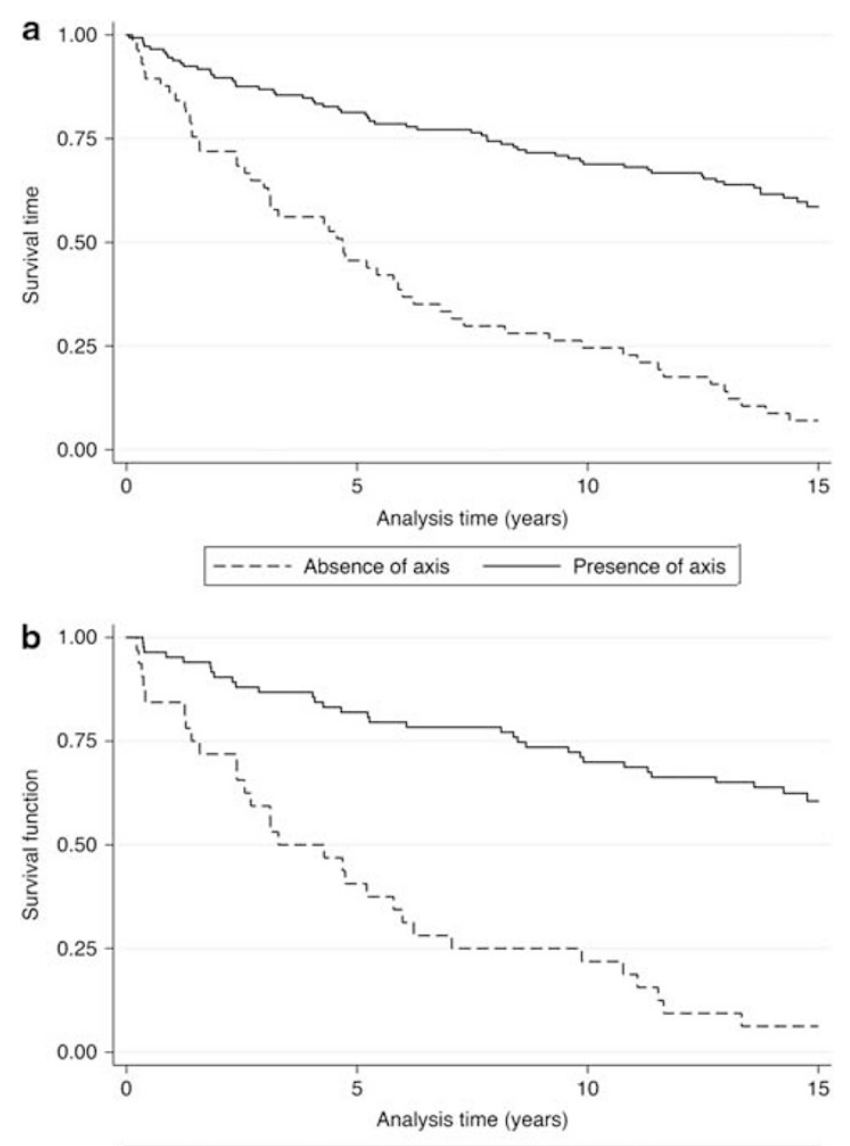

---- Absence of expression pattern

Figure 2 Kaplan-Meir survival curves for the presence of NTRK1pY674/pY675 together with the absence or low levels of PTPN6 and TP53 in estrogen receptor-positive and luminal A tumors. (a) Kaplan-Meir survival curve for estrogen receptor-positive tumors that express phosphorylated NTRK1 at Y674/Y675 together with lack or low levels of PTPN6. Tumors with this expression pattern (as represented by a solid black line) are associated with improved disease-free survival compared with samples where this pattern is absent (as represented by a dashed line). The separation is significant as identified by the Log-rank test $(P=0.024)$. (b) Kaplan-Meir survival curve for luminal A tumors (ER and PgR positive, HER2 negative) that express phosphorylated NTRK1 at Y674/Y675 together with lack or low levels of PTPN6. Tumors with this expression pattern (as represented with the solid black line) are associated with improved disease-free survival compared with samples where this pattern is absent (as represented by a dashed line). The separation is significant as identified by the Log-rank test $(P<0.001)$.

Prior examination of NTRK1 in breast cancer has revealed that nerve growth factor activates NTRK1 to promote cell proliferation, tumor growth, and metastasis. ${ }^{7,8,16}$ Paradoxically, NTRK1 mRNA has been shown to be associated with prolonged survival in breast cancer ${ }^{6}$ while tumors expressing nerve growth factor are a predictor of poor diseasefree survival. ${ }^{9,16}$ Given the contradictory nature of these results and that our previous findings demonstrate a ligand-independent mechanism of NTRK1-Y674/Y675 phosphorylation, via wild-type TP53-dependent repression of PTPN6 expression, leading to suppression of breast cancer cell proliferation, ${ }^{28}$ our laboratory has focused on investigating the presence of the members of this mechanism in breast cancer tumors.

We have demonstrated that NTRK1-pY674/pY675 was significantly and independently predictive of good 5-, 10-, and 15-year disease-free survival in a multivariate analysis that included PTPN6 and TP53, adjusted for ER, PgR, HER2, and Ki-67 expression, and nodal status. This suggests that the presence of NTRK1-pY675/pY675 together with low or lack of TP53 and PTPN6 levels is a predictor of improved disease-free survival. Importantly, NTRK1-pY674/pY675 did not show any significant association with disease-free survival in the univariate analysis (data not shown), which is indicative of the importance of the role of NTRK1-pY674/ pY675 in the context of TP53 and PTPN6. This is expected based on our previous finding regarding the relationship between NTRK1-pY674/pY675, PTPN6, and TP53 in breast cancer. ${ }^{28}$

Analysis of NTRK1-pY674/pY675 together with PTPN6 in 45 tumors with known wild-type TP53 genotype status showed a trend toward improved survival. However, the $P$-values were greater than 0.05 , which may reflect the low numbers of samples analyzed. Given that it has been revealed that estrogen receptor-positive tumors express wildtype TP53, ${ }^{45,56,57}$ we explored the expression of NTRK1-pY674/pY675 and PTPN6 in estrogen receptor-positive tumors. The predictive value of NTRK1-pY674/pY675 together with low levels of PTPN6 followed the same trends as the multivariate analysis of the unstratified data set. Furthermore, the presence of NTRK1-pY674/pY675 together with low or undetectable levels of PTPN6 was estimated, using the Kaplan-Meir method, to have a 59\% survival at 15 years' disease-free survival with a median survival time of 15 years. This compared with only $7 \%$ survival with a median survival time of 4.5 years when this expression pattern was absent (Figure 2a).

Recent attention has focused on classifying breast tumors by their hormone receptor and HER2 status, and molecular profile. ${ }^{55-58}$ Luminal A tumors are associated with wild-type TP53, low histological tumor grade, negative node, low-cell proliferation, and improved disease-free survival. ${ }^{55-58}$ Analysis of NTRK1-pY674/pY675 and low or undetectable levels of PTPN6 and TP53 in luminal A tumors, showed association with improved 15-year diseasefree survival by $79 \%$ in the multivariate analysis (Figure 2b). Moreover, Kaplan-Meir estimates demonstrated a $61 \%$ probability of 15 years' diseasefree survival with a median survival time exceeding 15 years compared with only a $6 \%$ probability of 15 years' disease-free survival and a median survival time of 3 years in its absence. Similar observations were seen in luminal B tumors. In this small subset of tumors, univariate analysis for NTRK1pY674/Y675 and low levels of TP53 and PTPN6 
Table 6 Multivariate analysis ${ }^{a}$ for luminal A tumors ${ }^{b}$ expressing phosphorylated NTRK1 at Y674/Y675 together with lack or low levels of TP53 and PTPN6.

\begin{tabular}{|c|c|c|c|c|c|c|}
\hline \multirow[b]{2}{*}{ Variable } & \multicolumn{2}{|l|}{ 5-year } & \multicolumn{2}{|l|}{ 10-year } & \multicolumn{2}{|l|}{ 15-year } \\
\hline & Hazard ratio (CI) & $\mathrm{P}$ & Hazard ratio (CI) & $\mathrm{P}$ & Hazard ratio (CI) & $\mathrm{P}$ \\
\hline \multicolumn{7}{|c|}{ NTRK1-pY674/pY675/PTPN6/TP53 mechanism } \\
\hline Fails & 1 & & 1 & & 1 & \\
\hline Meets ${ }^{\mathrm{C}}$ & $0.29(0.12-0.72)$ & 0.008 & $0.25(0.12-0.52)$ & $<\mathbf{0 . 0 0 1}$ & $0.21(0.11-0.41)$ & $<0.001$ \\
\hline
\end{tabular}

CI, confidence interval.

${ }^{\mathrm{a}}$ Multivariate analysis Cox regression model. 0, absence of expression; 1, weak expression; 2, moderate expression; 3, strong expression.

${ }^{b}$ Luminal A tumors, ER and PgR positive and HER2 negative.

${ }^{\mathrm{C}}$ Meets: Presence of phosphorylated NTRK1 at Y674/Y675 and absence or low expression of TP53 and PTPN6.

Bold values indicate significant $P$-values.

(Supplementary Table S6) revealed a trend toward improved 5 and 10 years' disease-free survival by $64 \%$ (hazard ratio $=0.36, P=0.081$ ) and $60 \%$ (hazard ratio $=0.40, P=0.058$ ), respectively. This expression pattern was associated with a $70 \%$ (hazard ratio $=0.30, P=0.007$ ) 15 years' disease-free survival. Kaplan-Meir estimates (Supplementary Figure S1) showed that luminal B tumors harboring this expression pattern were associated with a $51 \%$ probability of 15 years' disease-free survival and a median survival time of 14.5 years, compared with when it was absent in which there was association with a 35\% probability of 15 years' disease-free survival and a median survival time of 7 years.

Although the consensus in the p53 field is that low TP53 expression is associated with wild-type TP53, ${ }^{47-50}$ it is important to indicate that DNA of all tumor samples needs to be sequenced in order to correlate the presence of wild-type TP53 with PTPN6 and NTRK1-pY674/pY675 in our data set.

Yip et $a l^{26}$ have previously detected high PTPN6 $m R N A$ expression in primary breast cancer tumors. Insabato et $a l^{27}$ reported that PTPN6 protein expression correlates with grade III in $7 \%$ of breast tumors. ${ }^{27}$ This investigation has shown that PTPN6 overexpression was an independent predictor of poor 5,10 , and 15 years' disease-free survival in estrogen receptor-positive tumors with hazard ratios of $2.60 \quad(P=0.029), \quad 2.07 \quad(P=0.069), \quad$ and 2.13 $(P=0.040)$, respectively. These findings together with results showing a correlation between high PTPN6 levels and grade III by Pearsons $\chi^{2}$ (correlation $=0.153, \quad P=0.013$ ) and Spearman's rank (rank $=0.151, P=0.015$ ) support the findings of Insabato et al. ${ }^{27}$

This investigation has demonstrated that breast cancer tumors expressing NTRK1-pY674/pY675 together with low or undetectable levels of TP53 and PTPN6 are strongly associated with improved 5, 10 , and 15 years' disease-free survival. These observations support an important role for the mechanism of NTRK1-Y674/Y675 phosphorylation in suppressing breast cancer cell growth that our laboratory has previously identified. ${ }^{28}$ This potentially provides an attractive therapeutic target where a chemical compound that induces phosphorylation of NTRK1 at tyrosines 674/675 could be investigated for possible treatment, similarly to CPPy and gambogic amide, which have been shown to phosphorylate NTRK1 at Y490 and are possible candidates for the treatment of neuroblastoma and stroke, respectively. ${ }^{61,62}$ Given that activation of signal transduction pathways via NTRK1-pY674/pY675 involve the activation of the adaptors, SHB-2, APS GRB-2 and FRS-2, ${ }^{63-65}$ it will be important to address the transcriptomic and proteomic landscapes of tumors with this mechanism compared with tumors where this mechanism is absent. This question is currently being addressed by our group. Despite decades of research, breast cancer remains one of the most prominent causes of mortality for women in the world. The NTRK1-pY674/pY675, PTPN6, wildtype TP53 mechanism ${ }^{28}$ provides a potentially novel candidate for therapeutic intervention.

\section{Acknowledgements}

We thank Lars Holmberg and Fredrik Sandin for their valuable guidance and suggestions regarding the statistical analysis and the contents of this paper. We also thank Robert Springall for technical help regarding immunohistochemistry. A special thanks to David Bacon for technical help with photography and graphics.

\section{Disclosure/conflict of interest}

The authors declare no conflict of interest.

\section{References}

1 Jemal A, Bray F, Center MM, et al. Global cancer statistics. CA Cancer J Clin 2011;61:69-90.

2 Forouzanfar MH, Foreman KJ, Delossantos AM, et al. Breast and cervical cancer in 187 countries between 1980 and 2010: a systematic analysis. Lancet 2011;378:1461-1484. 
3 Harris R, Yeatts J, Kinsinger L. Breast cancer screening for women ages 50 to 69 years a systematic review of observational evidence. Prev Med 2011;53:108-114.

4 DeSantis C, Siegel R, Bandi P, et al. Breast cancer statistics, 2011. CA Cancer J Clin 2011;61:409-418.

5 Draper L. Breast cancer: trends, risks, treatments, and effects. AAOHN J 2006;54:445-451.

6 Descamps S, Pawlowski V, Revillion F, et al. Expression of nerve growth factor receptors and their prognostic value in human breast cancer. Cancer Res 2001;61:4337-4340.

7 Descamps S, Toillon RA, Adriaenssens E, et al. Nerve growth factor stimulates proliferation and survival of human breast cancer cells through two distinct signaling pathways. J Biol Chem 2001;276:17864-17870.

8 Lagadec C, Meignan S, Adriaenssens E, et al. TrkA overexpression enhances growth and metastasis of breast cancer cells. Oncogene 2009;28:1960-1970.

9 Sakamoto Y, Kitajima Y, Edakuni G, et al. Combined evaluation of NGF and p75NGFR expression is a biomarker for predicting prognosis in human invasive ductal breast carcinoma. Oncol Rep 2001;8:973-980.

10 Kumar S, Walia V, Ray M, et al. p53 in breast cancer: mutation and countermeasures. Front Biosci 2007;12:4168-4178.

11 Concin N, Zeillinger C, Tong D, et al. Comparison of p53 mutational status with mRNA and protein expression in a panel of 24 human breast carcinoma cell lines. Breast Cancer Res Treat 2003;79:37-46.

12 Petitjean A, Achatz MI, Borresen-Dale AL, et al. TP53 mutations in human cancers: functional selection and impact on cancer prognosis and outcomes. Oncogene 2007;26:2157-2165.

13 Walerych D, Napoli M, Collavin L, et al. The rebel angel: mutant p53 as the driving oncogene in breast cancer. Carcinogenesis 2012;33:2007-2017.

14 Thompson AM, Lane DP. p53 transcriptional pathways in breast cancer: the good, the bad and the complex. J Pathol 2010;220:401-403.

15 Reichardt LF. Neurotrophin-regulated signalling pathways. Philos Trans R Soc Lond B Biol Sci 2006;361: 1545-1564.

16 Dolle L, El Yazidi-Belkoura I, Adriaenssens E, et al. Nerve growth factor overexpression and autocrine loop in breast cancer cells. Oncogene 2003;22:5592-5601.

17 Vousden KH, Prives C. Blinded by the light: the growing complexity of p53. Cell 2009;137:413-431.

18 Beckerman R, Prives C. Transcriptional regulation by p53. Cold Spring Harb Perspect Biol 2010;2:a000935.

19 Imbriano C, Gurtner A, Cocchiarella F, et al. Direct p53 transcriptional repression: in vivo analysis of CCAATcontaining G2/M promoters. Mol Cell Biol 2005;25:3737-3751.

20 Stommel JM, Marchenko ND, Jimenez GS, et al. A leucine-rich nuclear export signal in the p53 tetramerization domain: regulation of subcellular localization and p53 activity by NES masking. EMBO J 1999;18:1660-1672.

21 Moll UM, Riou G, Levine AJ. Two distinct mechanisms alter p53 in breast cancer: mutation and nuclear exclusion. Proc Natl Acad Sci USA 1992;89: 7262-7266.

22 Green DR, Kroemer G. Cytoplasmic functions of the tumor suppressor p53. Nature 2009;458:1127-1130.

23 Mauldin IS, Tung KS, Lorenz UM. The tyrosine phosphatase SHP-1 dampens murine Th17 development. Blood 2012;119:4419-4429.
24 Lorenz U. SHP-1 and SHP-2 in T cells: two phosphatases functioning at many levels. Immunol Rev 2009;228:342-359.

25 Marsh HN, Dubreuil CI, Quevedo C, et al. SHP-1 negatively regulates neuronal survival by functioning as a TrkA phosphatase. J Cell Biol 2003;163:999-1010.

26 Yip SS, Crew AJ, Gee JM, et al. Up-regulation of the protein tyrosine phosphatase SHP-1 in human breast cancer and correlation with GRB2 expression. Int J Cancer 2000;88:363-368.

27 Insabato L, Amelio I, Quarto $\mathrm{M}$, et al. Elevated expression of the tyrosine phosphatase SHP-1 defines a subset of high-grade breast tumors. Oncology 2009;77:378-384.

28 Montano X. Repression of SHP-1 expression by p53 leads to trkA tyrosine phosphorylation and suppression of breast cancer cell proliferation. Oncogene 2009;28:3787-3800.

29 Dowsett M, Nielsen TO, A'Hern R, et al. Assessment of Ki67 in breast cancer: recommendations from the International Ki67 in Breast Cancer working group. J Natl Cancer Inst 2011;103:1656-1664.

30 Vojtesek B, Bartek J, Midgley CA, et al. An immunochemical analysis of the human nuclear phosphoprotein p53. New monoclonal antibodies and epitope mapping using recombinant p53. J Immunol Methods 1992;151:237-244.

31 Bartek J, Bartkova J, Lukas J, et al. Immunohistochemical analysis of the p53 oncoprotein on paraffin sections using a series of novel monoclonal antibodies. J Pathol 1993;169:27-34.

32 Brown A, Browes C, Mitchell M, et al. c-abl is involved in the association of p53 and trk A. Oncogene 2000;19:3032-3040.

33 Massa PT, Saha S, Wu C, et al. Expression and function of the protein tyrosine phosphatase SHP-1 in oligodendrocytes. Glia 2000;29:376-385.

34 Witkiewicz A, Raghunath P, Wasik A, et al. Loss of SHP-1 tyrosine phosphatase expression correlates with the advanced stages of cutaneous T-cell lymphoma. Hum Pathol 2007;38:462-467.

35 Pandey MK, Sung B, Ahn KS, et al. Butein suppresses constitutive and inducible signal transducer and activator of transcription (STAT) 3 activation and STAT3-regulated gene products through the induction of a protein tyrosine phosphatase SHP-1. Mol Pharmacol 2009;75:525-533.

36 Browes C, Rowe J, Brown A, et al. Analysis of trk A and p53 association. FEBS Lett 2001;497:20-25.

37 Kramer K, Gerald W, LeSauteur L, et al. Monoclonal antibody to human Trk-A: diagnostic and therapeutic potential in neuroblastoma. Eur J Cancer 1997;33: 2090-2091.

38 Moises T, Dreier A, Flohr S, et al. Tracking TrkA's trafficking: NGF receptor trafficking controls NGF receptor signaling. Mol Neurobiol 2007;35:151-159.

$39 \mathrm{Fu} \mathrm{X,} \mathrm{Zang} \mathrm{K,} \mathrm{Zhou} \mathrm{Z,} \mathrm{et} \mathrm{al.} \mathrm{Retrograde} \mathrm{neurotrophic}$ signaling requires a protein interacting with receptor tyrosine kinases via $\mathrm{C} 2 \mathrm{H} 2$ zinc fingers. Mol Biol Cell 2010;21:36-49.

40 Philippidou P, Valdez G, Akmentin W, et al. Trk retrograde signaling requires persistent, Pincher-directed endosomes. Proc Natl Acad Sci USA 2011;108: 852-857.

41 Lu W, Pochampally R, Chen L, et al. Nuclear exclusion of p53 in a subset of tumors requires MDM2 function. Oncogene 2000;19:232-240. 
42 Caleffi M, Teague MW, Jensen RA, et al. p53 gene mutations and steroid receptor status in breast cancer. Clinicopathologic correlations and prognostic assessment. Cancer 1994;73:2147-2156.

43 Yang LH, Tseng HS, Lin C, et al. Survival benefit of tamoxifen in estrogen receptor-negative and progesterone receptor-positive low-grade breast cancer patients. J Breast Cancer 2012;15:288-295.

44 Bardou VJ, Arpino G, Elledge RM, et al. Progesterone receptor status significantly improves outcome prediction over estrogen receptor status alone for adjuvant endocrine therapy in two large breast cancer databases. J Clin Oncol 2003;21:1973-1979.

45 Petrelli F, Barni S. Role of HER2-neu as a prognostic factor for survival and relapse in pT1a-bNoM0 breast cancer: a systematic review of the literature with a pooled-analysis. Med Oncol 2012;29:2586-2593.

46 Turpin E, Bieche I, Bertheau P, et al. Increased incidence of ERBB2 overexpression and TP53 mutation in inflammatory breast cancer. Oncogene 2002;21: 7593-7597.

47 Bartek J, Bartkova J, Vojtesek B, et al. Patterns of expression of the p53 tumor suppressor in human breast tissues and tumors in situ and in vitro. Int J Cancer 1990;46:839-844.

48 Soini Y, Paakko P, Nuorva K, et al. Comparative analysis of p53 protein immunoreactivity in prostatic, lung and breast carcinomas. Virchows Arch A Pathol Anat Histopathol 1992;421:223-228.

49 Alsner J, Jensen V, Kyndi $\mathrm{M}$, et al. A comparison between p53 accumulation determined by immunohistochemistry and TP53 mutations as prognostic variables in tumors from breast cancer patients. Acta Oncol 2008;47:600-607.

50 Borresen-Dale AL. TP53 and breast cancer. Hum Mutat 2003;21:292-300.

51 Soussi T, Beroud C. Significance of TP53 mutations in human cancer: a critical analysis of mutations at $\mathrm{CpG}$ dinucleotides. Hum Mutat 2003;21:192-200.

52 Olivier M, Langerod A, Carrieri P, et al. The clinical value of somatic TP53 gene mutations in 1,794 patients with breast cancer. Clin Cancer Res 2006;12: 1157-1167.

53 Elledge RM, Lock-Lim S, Allred DC, et al. p53 mutation and tamoxifen resistance in breast cancer. Clin Cancer Res 1995;1:1203-1208.
54 Berns EM, Foekens JA, Vossen R, et al. Complete sequencing of TP53 predicts poor response to systemic therapy of advanced breast cancer. Cancer Res 2000;60:2155-2162.

55 Prat A, Perou CM. Deconstructing the molecular portraits of breast cancer. Mol Oncol 2011;5:5-23.

56 Eroles P, Bosch A, Perez-Fidalgo JA, et al. Molecular biology in breast cancer: intrinsic subtypes and signaling pathways. Cancer Treat Rev 2012;38: 698-707.

57 Cancer Genome Atlas Network. Comprehensive molecular portraits of human breast tumors. Nature 2012;490:61-70.

58 Geyer FC, Rodrigues DN, Weigelt B, et al. Molecular classification of estrogen receptor-positive/luminal breast cancers. Adv Anat Pathol 2012;19:39-53.

59 Dumay A, Feugeas JP, Wittmer E, et al. Distinct tumor protein p53 mutants in breast cancer subgroups. Int J Cancer 2012;132:1227-1231.

60 Miller LD, Smeds J, George J, et al. An expression signature for p53 status in human breast cancer predicts mutation status, transcriptional effects, and patient survival. Proc Natl Acad Sci USA 2005;102: 13550-13555

61 Yamaguchi Y, Tabata K, Asami S, et al. A novel cyclophane compound, CPPy, facilitates NGF-induced TrkA signal transduction and induces cell differentiation in neuroblastoma. Biol Pharm Bull 2007;30: 638-643.

62 Jang SW, Okada M, Sayeed I, et al. Gambogic amide, a selective agonist for TrkA receptor that possesses robust neurotrophic activity, prevents neuronal cell death. Proc Natl Acad Sci USA 2007;104:16329-16334.

63 Qian X, Ginty DD. SH2-B and APS are multimeric adapters that augment TrkA signaling. Mol Cell Biol 2001;21:1613-1620.

64 MacDonald JI, Gryz EA, Kubu CJ, et al. Direct binding of the signaling adapter protein Grb2 to the activation loop tyrosines on the nerve growth factor receptor tyrosine kinase, TrkA. J Biol Chem 2000;275: 18225-18233.

65 Ong SH, Guy GR, Hadari YR, et al. FRS2 proteins recruit intracellular signaling pathways by binding to diverse targets on fibroblast growth factor and nerve growth factor receptors. Mol Cell Biol 2000;20: 979-989.

Supplementary Information accompanies the paper on Modern Pathology website (http://www.nature.com/ modpathol) 